\title{
A New Fractional Differential and Sobel Operator Based Edge Detection Method
}

\author{
Heng-tan QIU ${ }^{1, a}$, Xue-liang PING ${ }^{2, b}$, Jia-wei $\mathrm{He}^{3, \mathrm{c}}$ and Yong-zhong DU ${ }^{4, \mathrm{~d}}$ \\ ${ }^{1,2,3,4}$ School of mechanical engineering, Jiangnan University, Jiangsu Province Key Laboratory of \\ Advanced Food Manufacturing Equipment and Technology, Wuxi 214122, P. R. China

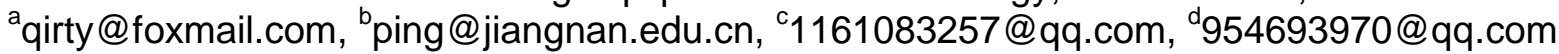

Keywords: edge detection; fractional differential; Sobel operator; texture details.

\begin{abstract}
Present edge detection algorithms are sensitive to noises, they may not get ideal effects on image edge detections and results in unclear image edges. To overcome these shortages, a new edge detection model based on the image edge detection method of Sobel operator and theory of fractional differential is proposed. Both theoretical researches and simulation results show that the new model can be used to extract more detail edge information than the classic method with a better anti-noise capability. It is an effective edge detection method.
\end{abstract}

\section{Introduction}

Edges, detail and texture features are the main image information which are of great significance for the visual effect of image and are very important to image analysis and understanding[1]. As an important content of digital image processing, edge detection extracts image edge contour and eliminates irrelevant information to reduce the amount of data analysis[2,3]. In the last decade, the use of fractional differential theory in image processing applications has been a new hotspot, and has been successfully applied to solve image processing problems such as image enhancement, image denoising , edge detection and so on [4-6].

JIANG Wei [4] combined the fractional-order differentiation theory with the existing Laplacian operator method, proposed a new image edge detection model based on fractional-order differentiation and compared with the existing integer-order differentiation edge detection methods. The experimental results showed that the model can detect the image edge well and have a certain effect on the noises. WANG Bin [5] proposed a new fractional order differential based on image edge detection operator which derives from the fractional order differential equation based on non-integer step according to the Riemann-Liouville definition and uses the method of Lagrange interpolation polynomial to get the gray value of non-integer step pixel. The method can extract more detail edge information than the classic one and has a better anti-noise capability. Zhang Jun [6] proposed a fractional variation image denoising model which can improve the peak signal to noise ratio of the noisy image and preserve more fine scale features in the process of denoising.

In this paper the fractional differential is combined with Sobel operator. It has been improved and applied to the image processing of edge detection. Ultimately, a new edge detection model is put forward. These experiments show that the model can not only extract the image edge features, but also have a good noise inhibition. The model can detect more image texture details, which is better than the commonly used integer order differential edge detection algorithm. It is an effective and superior edge detection processing method for texture detail-rich images.

\section{A new model for edge detection}

By increase of differential order, the fractional differential gains a sharp nonlinear growth and also weakens the low frequency signal. The second order differentiation gains more increase in the high frequency signal than differential spectrum, and performs better on weakening low frequency signal than differential spectrum. To the fractional differential, the increased level of high frequency signal is a little weaker than differential spectrum and the second order differentiation, but it has achieved a 
satisfied increase and the intermediate frequency signal is strengthened. While the low frequency signal keeps retaining-nonlinearity instead of suffering sharp decrease. The application of fractional differential to edge detection can not only collect the information of image edge but also keep texture detailed information which changes little in gray in smooth area of the image. It also has good performance in the restricting noise.

Sobel operator performs a 2-D spatial gradient measurement on an image. Its gradient magnitude is consistent along different direction when using Sobel edge detection algorithm detecting edge. The corresponding horizontal and vertical gradient operators of Sobel algorithm are as follows:

$$
S_{R}=\frac{1}{4}\left[\begin{array}{ccc}
1 & 0 & -1 \\
2 & 0 & -2 \\
1 & 0 & -1
\end{array}\right], S_{C}=\frac{1}{4}\left[\begin{array}{ccc}
-1 & -2 & -1 \\
0 & 0 & 0 \\
1 & 2 & 1
\end{array}\right]
$$

Let $F(x, y)$ as the gradation function of an image. The pixels of a $3 \times 3$ image are aligned as follows:

$$
\begin{array}{|ccc|}
F(x-1, y+1) & F(x, y+1) & F(x+1, y+1) \\
F(x-1, y) & F(x, y) & F(x+1, y) \\
F(x-1, y-1) & F(x, y-1) & F(x+1, y-1) \\
\hline
\end{array}
$$

Fig. $13 \times 3$ neighborhood pixels

The horizontal and the vertical gradients can be obtained from this pixel (x,y) area using convolution masks. They are depicted as follows:

$$
\begin{aligned}
& G_{R}(x, y)=\frac{1}{4}[F(x-1, y+1)-F(x+1, y+1)+2 F(x-1, y)-2 F(x+1, y)+F(x-1, y-1)-F(x+1, y-1)] \\
& G_{C}(x, y)=\frac{1}{4}[F(x-1, y-1)-F(x-1, y+1)+2 F(x, y-1)-2 F(x, y+1)+F(x+1, y-1)-F(x+1, y+1)]
\end{aligned}
$$

Where, $G_{R}(x, y)$ denotes the horizontal gradient, and $G_{C}(x, y)$ the vertical gradient. Then do equal interval sampling by taking steps $\Delta x=2$, we can get

$$
\left\{\begin{array}{c}
F(x-1, y+1)-F(x+1, y+1)=-2 \frac{\partial F(x+1, y+1)}{\partial x} \\
F(x-1, y)-F(x+1, y)=-2 \frac{\partial F(x+1, y)}{\partial x} \\
F(x-1, y-1)-F(x+1, y-1)=-2 \frac{\partial F(x+1, y-1)}{\partial x}
\end{array}\right.
$$

Therefore, the differential of $G_{R}(x, y)$ and $G_{C}(x, y)$ can be expressed as:

$$
\begin{aligned}
& G_{R}(x, y)=-\frac{1}{2}\left(\frac{\partial F(x+1, y+1)}{\partial x}+2 \frac{\partial F(x+1, y)}{\partial x}+\frac{\partial F(x+1, y-1)}{\partial x}\right) \\
& G_{C}(x, y)=-\frac{1}{2}\left(\frac{\partial F(x-1, y+1)}{\partial x}+2 \frac{\partial F(x, y+1)}{\partial x}+\frac{\partial F(x+1, y+1)}{\partial x}\right)
\end{aligned}
$$

Replace the integer-order differentials with the fractional differentials in Eq. (4) and Eq. (5), then we get the new established model:

$$
\left\{\begin{array}{l}
G_{R}^{V}(x, y)=\frac{1}{2}\left[\frac{\partial^{V} F(x+1, y+1)}{\partial x^{V}}+2 \frac{\partial^{V} F(x+1, y)}{\partial x^{V}}+\frac{\partial^{V} F(x+1, y-1)}{\partial x^{V}}\right] \\
G_{C}^{V}(x, y)=\frac{1}{2}\left[\frac{\partial^{V} F(x-1, y+1)}{\partial x^{V}}+2 \frac{\partial^{V} F(x, y+1)}{\partial x^{V}}+\frac{\partial^{V} F(x+1, y+1)}{\partial x^{V}}\right]
\end{array}\right.
$$

Where $G_{R}(x, y)$ denotes the improved horizontal gradient and $G_{C}(x, y)$ the vertical gradient. In order to achieve a better edge detection, take the former three items of the fractional differential expression in $F(x, y)$ :

$$
\left\{\begin{array}{l}
\frac{\partial^{V} F(x+1, y+1)}{\partial x^{V}} \approx F(x+1, \mathrm{y}+1)+(-v) F(x, y+1)+\frac{(-v)(-v+1)}{2} F(x-1, y+1) \\
\quad \frac{\partial^{V} F(x+1, y)}{\partial x^{V}} \approx F(x+1, \mathrm{y})+(-v) F(x, y)+\frac{(-v)(-v+1)}{2} F(x-1, y) \\
\frac{\partial^{V} F(x+1, y-1)}{\partial x^{V}} \approx F(x+1, \mathrm{y}-1)+(-v) F(x, y-1)+\frac{(-v)(-v+1)}{2} F(x-1, y-1)
\end{array}\right.
$$


Combined with Eq. (6) and Eq. (7), the horizontal and vertical gradient models of operator based on the Sobel operator and the fractional differential can be expressed as follows:

$$
\begin{aligned}
& G_{R}^{V}=-\frac{1}{2}\{F(x+1, y+1)+2 F(x+1, y)+F(x+1, y-1)+(-v)[F(x, y+1)+2 F(x, y)+F(x, y-1)]+ \\
& \left.\frac{v^{2}-v}{2}[F(x-1, y+1)+2 F(x-1, y)+F(x-1, y-1)]\right\} \\
& G_{R}^{V}=-\frac{1}{2}\{F(x-1, y+1)+2 F(x, y+1)+F(x+1, y+1)+(-v)[F(x-1, y)+2 F(x, y)+F(x+1, y)]+ \\
& \left.\frac{v^{2}-v}{2}[F(x-1, y-1)+2 F(x, y-1)+F(x+1, y-1)]\right\}
\end{aligned}
$$

The horizontal and vertical gradient operators of the improved model are as follows:

$$
S_{R}^{V}=\frac{1}{2}\left|\begin{array}{ccc}
\frac{v-v^{2}}{2} & v & -1 \\
v-v^{2} & 2 v & -2 \\
\frac{v-v^{2}}{2} & v & -1
\end{array}\right|, S_{C}^{V}=\frac{1}{2}\left|\begin{array}{ccc}
-1 & -2 & -1 \\
v & 2 v & v \\
\frac{v-v^{2}}{2} & v-v^{2} & \frac{v-v^{2}}{2}
\end{array}\right|
$$

Here, the selection of parameter $v$ plays an important role in extracting the edge detail and texture.

\section{Simulation experiment}

To the rice and sausage cases, use the MATLAB 7.0 to do simulation experiments based on this new model, and then compare with the results of the existing edge detection operator [9-10].

In the first group experiment, we test the result of the proposed method on rice image by comparing with the exiting primary methods of the edge detection. Fig. 2 shows the comparison of the edge extraction. Where Fig. 2(b)-(f) are respectively results of Sobel operator, Prewitt operator, Roberts operator, Canny operator and the proposed method $(T=50, v=0.7)$.

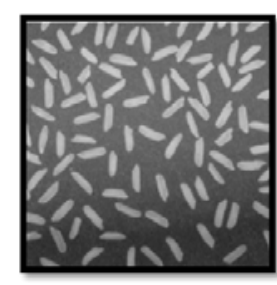

(a) original image



(b) Sobel

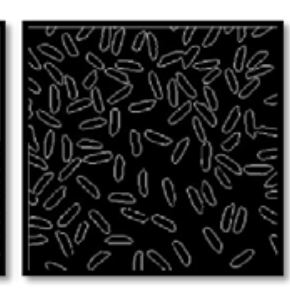

(c) Prewitt

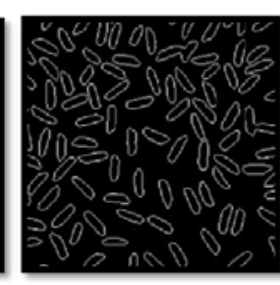

d) Roberts

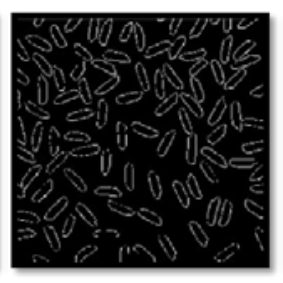

(e) Canny

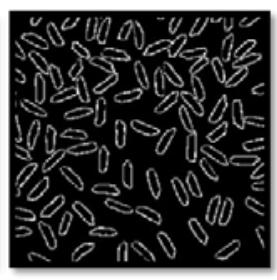

(f) the proposed method

Fig. 2 Rice image after processing with different methods

In the second group experiment, we illustrate the superiority of the new model compared with the existing edge detection methods. In terms of the texture detail-rich images, the proposed model can detect more texture details. Fig. 3 shows the experimental results.

Fig. 2 and Fig. 3 show that Sobel and Prewitt operators can both detect the edge. But when handling some points, they cannot make a strictly distinction between the main part of the image and its background. Roberts operator can also detect edges. But discontinuity of the edge would be found during the operation. Canny operator is an ideal method of edge detection. While getting a better detective result, it requires a larger filtering amount which may cause a loss of more detailed information. While, this new model can detect the edge more exactly and get more detailed information about the target object. With this new model, we can get more detective results comparing to those common edge detection methods and get close to the goal of edge detection.

Referring to the fractional differential theory, this paper set up a new model of the edge detection based on Sobel operator and fractional differential. On one hand, it keeps the advantage of Sobel operator in smoothing noise and removing parts of false edges. On the other hand, it keeps the characteristic of fractional differential that can detect texture detail of the image much well and get close to the goal of the edge detection. When it comes to the edge detection, this new model is 
superior to the existing Sobel operator edge detective methods and the fractional differential methods.

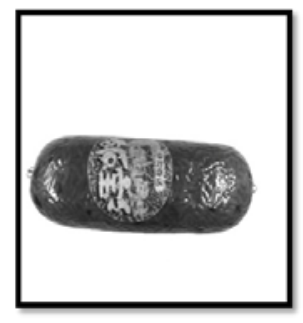

(a) original image

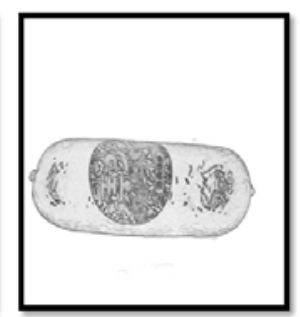

(b) Sobel

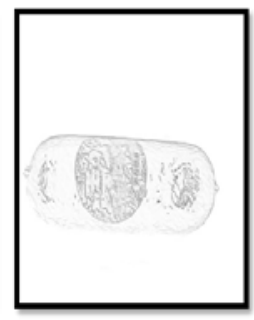

(c) Prewitt



d) Roberts
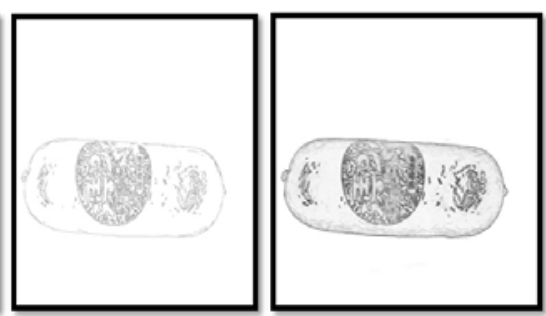

Fig. 3 Sausage image after processing with different methods

\section{Conclusion}

Referring to the fractional differential theory, this paper provides a new model based on the fractional differential and the edge detection of Sobel operator. This model can remedy the shortage of the existing edge detection. Through the comparison experiment to the existing edge detection method, this new model has a priority over the exiting edge detection method. It is an effective method about the edge detection that it can test more information of the image texture detail and also have good performance in the restricting noise. We should have more research on approaching to a more proper differential order in order to gain a more dependable test result.

\section{Reference}

[1] Mark S. Nixon, Alberto S. Aguado. Feature Extraction and Image Processing (second edition)[M].Beijing: Publishing house of electronic industry, 2010.10

[2] Deng G. Differentiation-based edge detection using the logarithmic image processing model. Mathematical Imaging and Vision, Vol.8, No.2 ,1998, pp.161-180.

[3] PENG Hong-Jing, CHEN Song-Can, ZHANG Dao-Qiang. A Local Learning Approach for Natural Image Matting. Journal of Software, Vol.20, No.4, April 2009, pp.834-844

[4] JIANG Wei, DING Zhi-quan, LIU Ya-wei. New image edge detection model based on fractional-order partial differention. Journal of Computer Applications,Vol.32,No.10, Oct. 2012, pp.2848-2858.

[5] WANG Bin, PU Yi-fei, ZHOU Ji-liu. New fractional order differential based image edge detection operator. Application Research of Computers,Vol.29,No.8,Aug. 2012,pp.3160-3162.

[6] ZHANG Jun, WEI Zhi -hui. Fractional variational model and projection algorithm for image denoising. Computer Engineering and Applications, Vol.45,No.5, 2008,pp.1-6.

[7] WANG Wei-xing, YU Xin. IAI Jun. Image enhancement for rock fractures based on fractional differential. Journal of Computer Applications, Vol.29,No.1l,Nov. 2009,pp. 3105-3107

[8] ZHANG Xu-xiu, LU Yang. Medical Image Edge Detection Method Based on Fractional Differential Coefficient. Journal of DaLian Jiaotong Uinversity. Vol.30, No.6, 2009, pp.61-65

[9] LUO Jun-hui, FENG Ping.MATLAB7.0 applications in image processing [M].Beijing:

Mechanical Industry Press, 2005.

[10]Gonzalez, RC, Woods, RE, \& Eddins, SL (2006). Gonzalez Digital Image Processing. Beijing: Electronic Industry Press, 2006. 